\title{
REDUCING THE EFFECTS OF FLOODING USING LOCAL INTELLIGENT SYSTEMS
}

\author{
PhD Eng. Mihai Mărgăritescu ${ }^{1}$, \\ Eng. Ana Maria Eulampia Rolea ${ }^{2}$, \\ PhD Student Eng. Anghel Constantin ${ }^{3}$ \\ 1,2,3 National Institute of Research and Development in Mechatronics \\ and Measurement Technique \\ Bucharest, Sos. Pantelimon 6-8, Romania \\ mihai.margaritescu@gmail.com, eulampia46@yahoo.com, anghel.constantin@incdmtm.ro
}

\begin{abstract}
Although various types of flood warning systems, which can be considered lesser or greater "smart", are already functional, however they are dependent of other complex systems, such as satellites, sensor networks or internet connection, which in certain situations could be not available. This project proposes a local intelligent system for the flooding anticipation, based on a collection of sensors and intelligent algorithms of data processing - independent or complementary to other existing warning system.
\end{abstract}

Keywords: Flood, Warning System, Intelligent System, Data Processing, Fuzzy Logic.

\section{Introduction}

Floods are natural disasters or are caused by human activities: Floods on rivers, as a result of heavy rainfall or sudden melting snow which exceed the capacity of the basin to receive the excessive amounts of water. Flooding can also occur if the water-course is blocked due to landslide mud, vegetation due to human activities. Flash flooding may occur in the case of uncontrolled discharge or the collapse of dams or reservoirs dams. Coastal floods are caused by storms or tsunamis that generate in-crease in sea level and flooding of coastal estuaries or rivers.

The most known systems are the so-named Expert systems, which are a branch of the Artificial Intelligence (AI), that use knowledge from a certain domain in order to solve a problem at the level of a human expert $[1,2,3]$. Such a system represents more than a classical programme because it incorporates the knowledge base, inference methods and decision assistance. The main types of flood warning systems are:

Sensor networks, such as networks of wireless sensors working in the $900 \mathrm{MHz}$ band acting in range of $8 \mathrm{~km}$ or on $144 \mathrm{Mhz}$ in range of $25 \mathrm{~km}$;

Warning systems based on Web. Data are real time collected from observation hydrologic stations by a cluster of servers and then the results are available on client computers;
RS (Remote Sensing) combined with GIS (Geographic Information System). Com-pare the satellite images obtained in real time with digital map databases.

Many of these systems are based on pattern recognition programs. In turn, some of the best systems of pattern recognition are based on neural networks - $[4,5]$;

Warning systems based on Radio, SMS, TV, telephony. Because the information received from sensors must be converted into a collection of decisions which are then recommended (suggested) to the human operator and the numerical expression sometimes is not the best solution, in practice are frequently used Fuzzy logic algorithms, operating with values that are closer to the verbal expression $[6,7]$

All these systems are complex and depending of other more complex systems, even global systems. This project proposes a small, cheap, independent and local system, composed of a sensory system placed on a Floating Vehicle (FV) and a Monitoring and Control Centre (MCC), communicating wireless each other. The project focuses on the sensory system and data processing, not on the floating vehicle itself. The raw data are processed in the MCC by the aid of various algorithms, the results consisting in warnings of certain degrees of danger. The MCC can be primarily directly accessible to the decision makers and secondly to a larger interest group via Internet. 


\section{System Architecture}

Information and ideas above are materialized in a block diagram depicting the system architecture, shown in Figure 1:

The central element of the module located in the FV is the data acquisition and control board, which acquires analogue or digital signals from sensors.

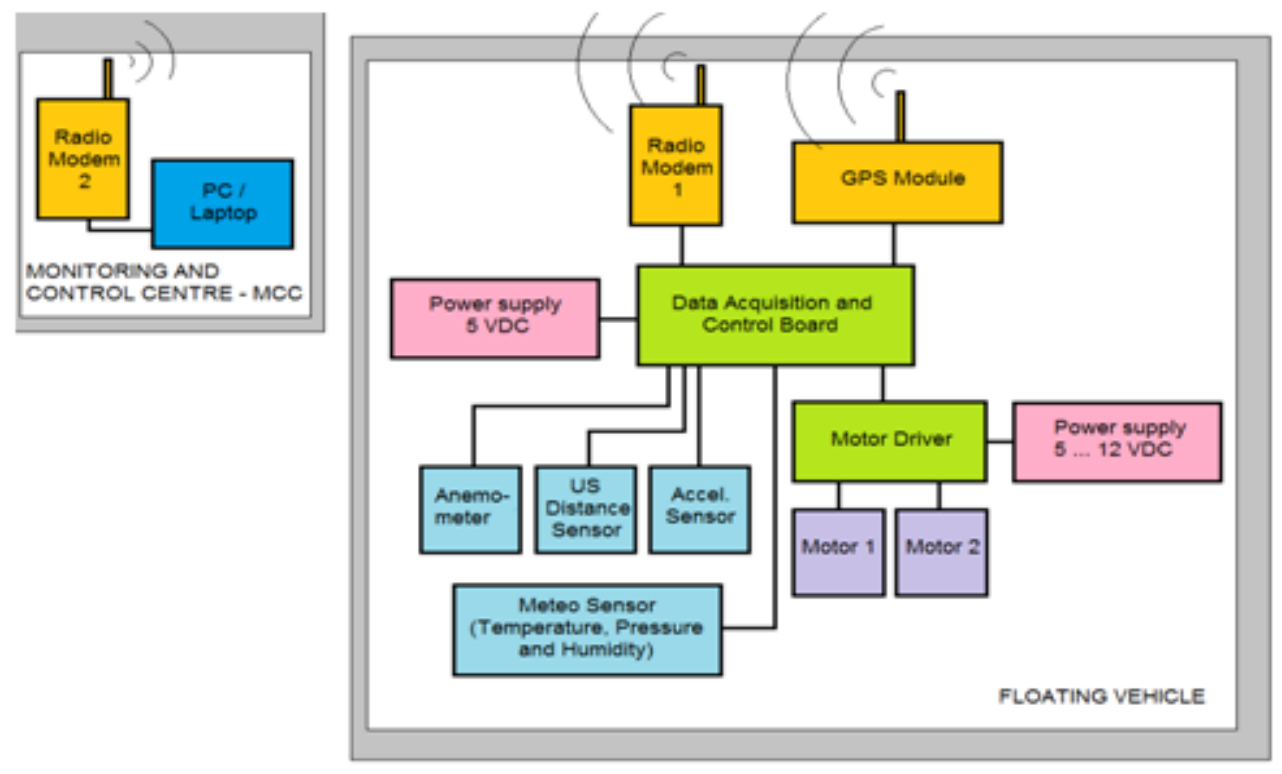

Figure 1: System architecture

In a first approach, the following sensors are used:

- a distance ultrasonic sensor which measures the vertical distance between the $\mathrm{FV}$ and a fixed reference on the bank, where it is anchored to the shore; This sensor provides information on the magnitude of the waves or if there are sudden changes in water level;

- an acceleration sensor that provides information about the vertical movement of the vehicle regardless of its position, whether or not anchored to the shore. In addition, it makes possible highlighting of the shocks which may be related to different natural phenomena;

- a combined "atmospheric" sensor, containing a temperature, pressure and humidity sensor that provides specific information;

- an anemometer which measures the wind speed.

Other sensors, such as an underwater sensor for temperature, pressure and turbidity measurement, a triaxial accelerometer or an underwater ultrasonic sensor can be subsequent added. The GPS module shows the position coordinates of the FV (latitude and longitude).

Another function of the controller is transmitting control signals to the two electric motors used for the FV propulsion and directing, via a driver - motors interface. This can be done in two ways:

- using a single motor for propulsion and the other for operating the rudder;
- the use of both motors for propulsion and directing, resulting from the combination of the speeds.

The transmission of the obtained data between FV and MCC is made through a pair of radio modems, working at the frequency of $2,4 \mathrm{GHz}$, which does not require licence in Romania and allows data transmission on distances up to several hundreds of meters. The data are received on a PC or terrain laptop, processed and presented by the aid of the application software.

\section{Sensory System Implementation}

The figure below shows the integrated sensory system with the following elements:

1. Data acquisition and control board Arduino Mega 2560 v3

2. Support plate

3. Connection module (Breadboard)

4. LiPo Battery $5200 \mathrm{mAh} / 7.4 \mathrm{~V}$

5. The ultrasonic distance sensor ping Paralax

6. Accelerometer ADXL 193

7. Atmospheric sensor (includes pressure, humidity and temperature sensors) BME280

8. Anemometer (including the 9 VDC power supply)

9. GPS Module

10. Wireless Wixel module USART connected to the data acquisition board

11. Wixel Wireless module USB connected to PC. 


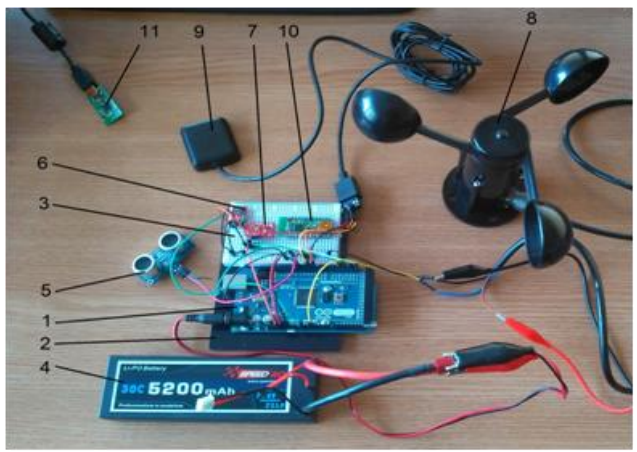

Figure 2: Sensory system

To obtain a graphical representation of the measured values it was used the free "PLX-DAQ" software [8] that connects the Arduino software and Microsoft Excel. The following figure shows the dynamically display of data in an Excel spreadsheet.
The data from the six sensors are wirelessly transmitted from the microcontroller to PC and real time graphically displayed - Figure 3:

\begin{tabular}{|c|c|c|c|c|c|c|}
\hline Time & $\begin{array}{l}\text { vitv } \\
{[\mathrm{m} / \mathrm{s}]}\end{array}$ & $\begin{array}{c}\text { acc } \\
{[\mathrm{m} / \mathrm{s} 2]}\end{array}$ & $\begin{array}{l}\text { dist } \\
{[\mathrm{cm}]}\end{array}$ & $\begin{array}{l}\text { temp } \\
\text { [grC] }\end{array}$ & $\begin{array}{c}\text { pres } \\
{[\mathrm{Pa} / 10000]}\end{array}$ & $\begin{array}{l}\text { umid } \\
\text { [RH\%] }\end{array}$ \\
\hline 15:19:37 & -0.16 & -10.55 & 165 & 26.39 & 10.07 & 38 \\
\hline 15:19:38 & -0.16 & -11.16 & 165 & 26.39 & 10.07 & 38 \\
\hline $15: 19: 38$ & -0.16 & -11.16 & 165 & 26.39 & 10.07 & 38 \\
\hline 15:19:39 & -0.16 & -11.16 & 165 & 26.39 & 10.07 & 38 \\
\hline 15:19:39 & -0.16 & -10.55 & 165 & 26.38 & 10.07 & 38 \\
\hline 15:19:40 & -0.16 & -10.55 & 165 & 26.39 & 10.07 & 38 \\
\hline $15: 19: 40$ & -0.16 & -8.11 & 165 & 26.39 & 10.07 & 38 \\
\hline $15: 19: 41$ & -0.16 & -8.72 & 165 & 26.39 & 10.07 & 38 \\
\hline $15: 19: 41$ & -0.16 & -10.55 & 165 & 26.38 & 10.07 & 38 \\
\hline $15: 19: 42$ & -0.16 & -11.16 & 165 & 26.4 & 10.07 & 38 \\
\hline 15:19:42 & -0.16 & -11.16 & 165 & 26.39 & 10.07 & 38 \\
\hline $15: 19: 43$ & 1.52 & -7.5 & 165 & 26.4 & 10.07 & 38 \\
\hline $15: 19: 43$ & 4.58 & -2 & 165 & 26.4 & 10.07 & 38 \\
\hline $15: 19: 44$ & 3.97 & -3.22 & 165 & 26.4 & 10.07 & 38 \\
\hline $15: 19: 44$ & 3.36 & 1.06 & 12 & 26.4 & 10.07 & 38 \\
\hline 15:19:45 & 2.90 & 45.04 & 31 & 26.4 & 10.07 & 40 \\
\hline $15: 19: 45$ & 2.59 & -11.16 & 165 & 26.4 & 10.07 & 38 \\
\hline $15: 19: 46$ & 2.29 & -14.83 & 165 & 26.41 & 10.07 & 38 \\
\hline $15: 19: 46$ & 2.14 & 20.61 & 165 & 26.42 & 10.07 & 39 \\
\hline 15:19:47 & 1.83 & -6.89 & 164 & 26.39 & 10.07 & 39 \\
\hline 15:19:47 & 1.68 & 20 & 164 & 26.4 & 10.07 & 39 \\
\hline $15: 19: 48$ & 1.52 & -25.83 & 164 & 26.4 & 10.07 & 39 \\
\hline $15: 19: 49$ & 1.37 & 8.39 & 165 & 26.4 & 10.07 & 39 \\
\hline $15: 19: 49$ & 1.37 & -51.49 & 165 & 26.41 & 10.07 & 39 \\
\hline 15:19:50 & 1.22 & -16.05 & 165 & 26.4 & 10.07 & 39 \\
\hline 15:19:50 & 1.22 & -6.89 & 165 & 26.4 & 10.07 & 39 \\
\hline $15: 19: 51$ & 0.91 & 6.55 & 27 & 26.41 & 10.07 & 39 \\
\hline 15:19:51 & 0.91 & -3.83 & 165 & 26.41 & 10.07 & 39 \\
\hline 15:19:52 & 0.91 & -8.11 & 165 & 26.96 & 10.07 & 45 \\
\hline 15:19:52 & 0.91 & -13 & 33 & 27.87 & 10.05 & 56 \\
\hline $15: 19: 53$ & 0.76 & -11.16 & 165 & 27.29 & 10.08 & 61 \\
\hline $15: 19: 53$ & 0.76 & -9.94 & 165 & 27.06 & 10.07 & 64 \\
\hline $15: 19: 54$ & 0.76 & -9.33 & 165 & 27.02 & 10.07 & 66 \\
\hline
\end{tabular}

Figure 3: Dynamically displaying data in an Excel file

The US distance sensor can prove usefulness in the event of a flood, at least for the downstream areas.

The model described above uses an US senzor working in atmosphere, but an underwater US senzor which measures the distance between FV and the river bottom or the seabed would be more useful [9].

Figure 4 presents the simulation of a flood, with stacked graphics of the raw data and the filtered data in order to remove the influence of waves:

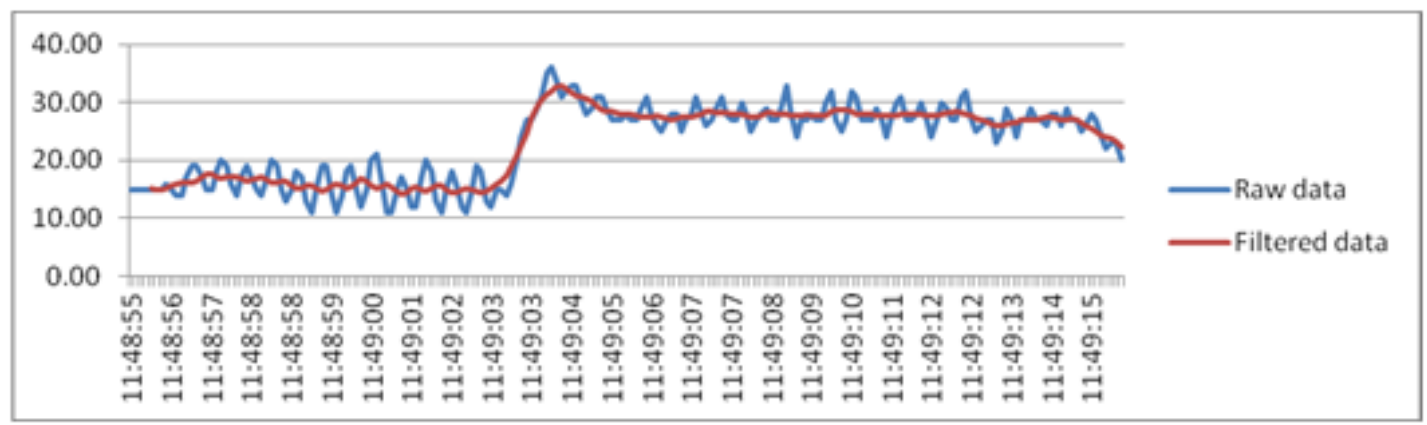

Figure 4: Image of a flood with simulated data 


\section{Use of the Fuzzy Model Mamdani Type for the Flood Warning}

In the previous paragraph was shown how data were obtained from several sensors, including an anemometer and a pressure sensor, hence pairs of values [vitv (wind speed), pressure].

They are introduced in the Matlab programme of FIS (Fuzzy Inference System) type [10], which models the flooding phenomenon on the basis of information from the sensors mentioned above. Thus is obtained a local monitoring system based on two sensors providing information that can predict the rain, and thus to some extent associated flooding, although the phenomenon is very complex and associated with geography of that zone and other factors. It is known that a low atmospheric pressure or its rapid decline is associated with producing of rainfall with an intensity according to a more or less proportional law.

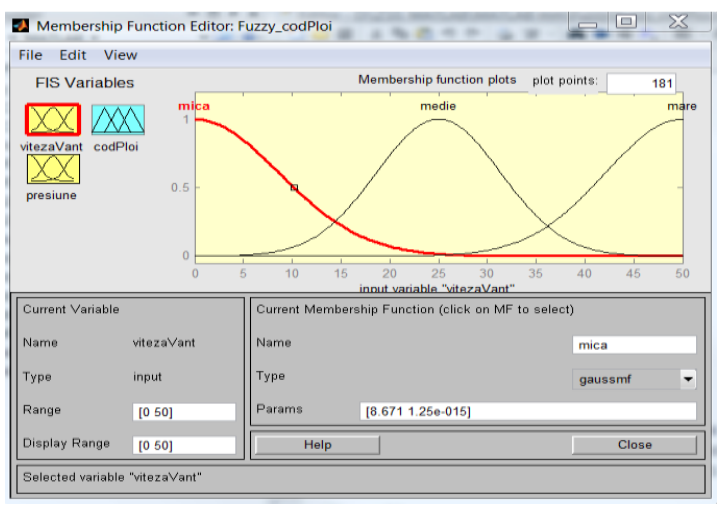

Figure 5: Mamdani editor of membership functions for wind speed
Also, an increased wind speed means changes in the weather; associated with a decrease of pressure may indicate a greater immediacy of precipitation. Therefore, the fusion of the information from an atmospheric pressure (barometric) sensor and one that measures the wind speed, and based on knowledge of meteorology, it can be estimated the precipitation probability of a certain intensity.

In the following it will be presented the process modelling of the precipitation provision based on data from the two sensors. Output linguistic values are expressed in the form of well-known precipitation codes - blue (no or very low risk), yellow (medium risk), orange (high risk) and red (very high risk). The Mamdani Fuzzy Inference System (FIS) editor allows the selection of the type of membership functions and their parameters, e.g. for the input variable "wind speed" Gaussian membership functions - Figure 5, respective for the output variable "code for precipitation" - of triangular type, Figure 6.

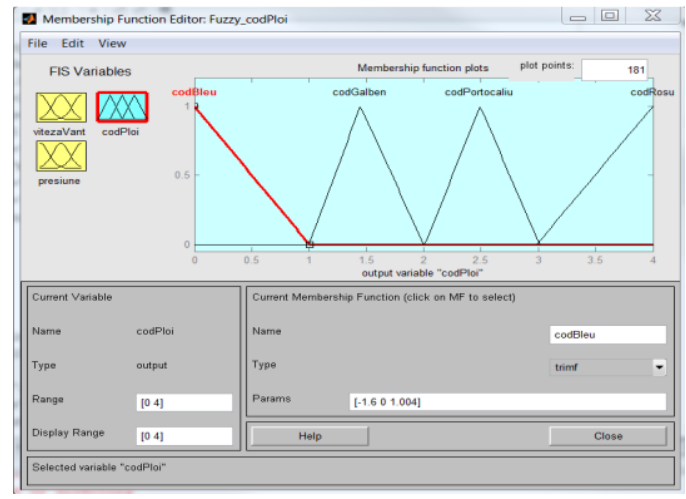

Figure 6: Mamdani editor of the membership functions for the output variable

The rules underlying the inference process are presented in the following table:

Table 1. The set of rules used in Mamdani FIS

\begin{tabular}{cccc}
\hline $\begin{array}{c}\text { Pressure / Wind } \\
\text { speed }\end{array}$ & Small & Medium & High \\
\hline Normal & Blue code & Blue code & Yellow code \\
Low & Blue code & Yellow code & Orange code \\
Very low & Yellow code & Orange code & Red code \\
\hline
\end{tabular}

Their implementation is made with the editor of rules, selecting "AND" option and using Table 1 which contains the nine fuzzy rules governing the system.

The fuzzy inference result is obtained by placing the two input values at the lower left of the panel (Input); in this case the two scales were chosen close to reality (from 0 to $50 \mathrm{~m} / \mathrm{s}$ for wind speed and the pressure 930-1050 hPa).

Figures 7 and 8 show the fuzzy output values (precipitation codes) for different input values with the defuzzyficated values indicated in the upper right corner of each panel. 


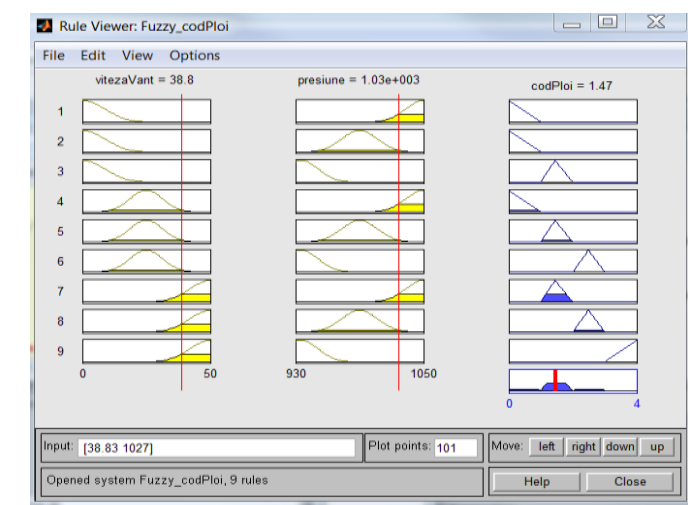

Figure7: Medium risk - Yellow code / output value 1.47

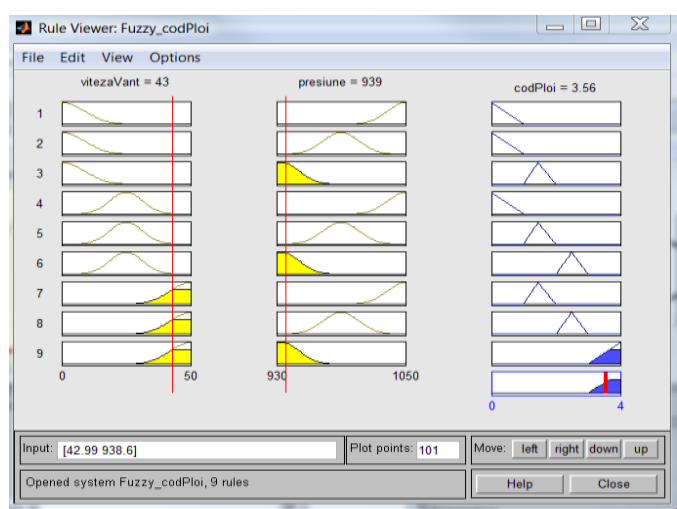

Figure 8: Very high risk - Red code / output value 3.56

Finally, the panel from Figure 9 shows a spatial representation of the risk level on the basis of the two measured parameters:

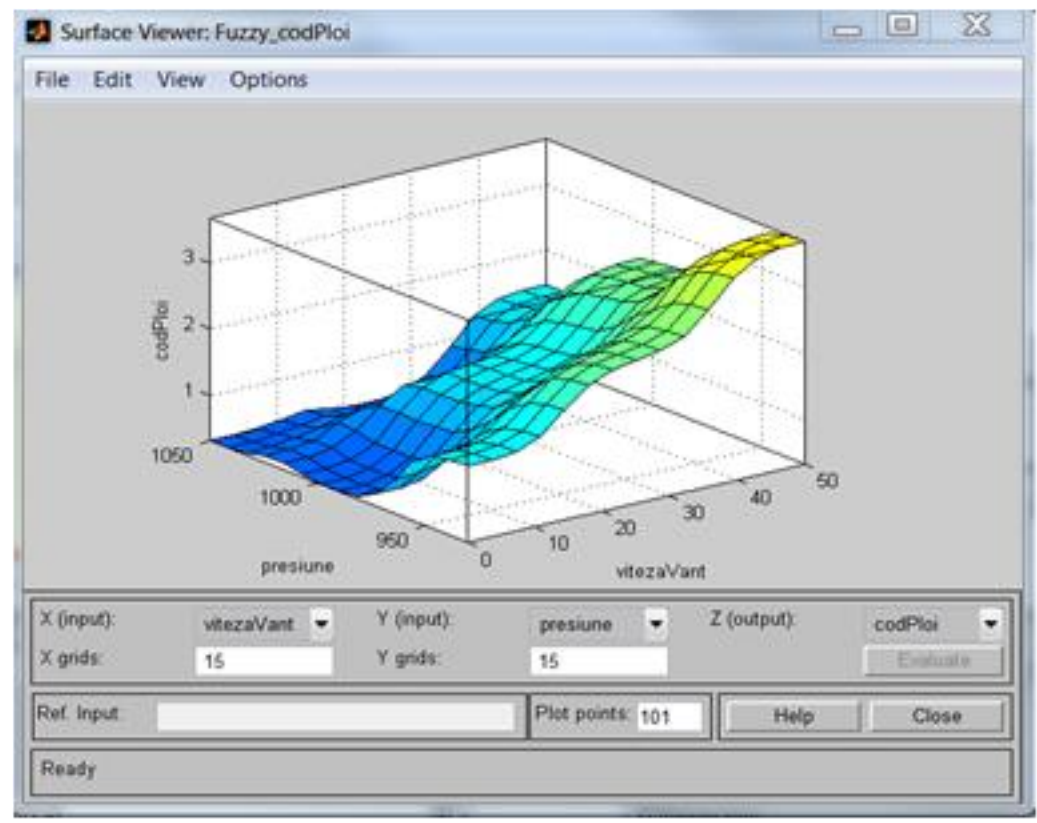

Figure 9: Mamdani editor associated with the risk of surface

In the fuzzy systems described above, the user chooses the membership functions and their parameters according to his intuition and experience, sometimes leading to questionable results.

To improve the inference system performance, were developed complex systems depending on the input data and expected results by specific adaptive neural network algorithms, calculating parameters that lead to the best results.

This requires a set of training input data and output data - the expected results. Using the method of back propagation or the method of least squares, or a combination thereof, the parameters of the membership functions are more convenient estimated [11].
Further work will concentrate to incorporate such advanced algorithms.

\section{Conclusions}

The model developed in this stage proved its functionality and can be extended especially with underwater sensors. The middle project results, based mainly on simulated data, constitute the basis of more complex programs, which use data from multiple sensors and deeper knowledge of meteorology, completing thus the image of the risk associated with the flooding events.

Finally, it is expected to obtain a small, cheap and local intelligent system, independent or complementary to other existing warning systems. 


\section{Acknowledgements}

This work was supported by a grant within the NUCLEU National Research Program, Ctr. 3N/2016, project number PN 16210102.

\section{References}

[1] Hsu-Yang Kung, Chi-Hua Chen, Hao-Hsiang Ku.: Designing intelligent disaster prediction models and systems for debris-flow. Expert Systems with Applications 39, (2012).

[2] Chen, Wang-Kun., Sui, GuangJun., DangL ing, Tang.: A Fuzzy Intelligent Decision Support System for Typhoon Disaster Management. IEEE International Conference on Fuzzy Sys-tems, Taiwan, (2011).

[3] Keoduangsine, S., Goodwin, R.: An Appropriate Flood Warning System in the Context of Developing Countries. International Journal of Innovation, Management and Technology, Vol. 3, No. 3, (2012).
[4] Enăchescu, C.: Calculul neuronal, (2008).

[5] Dioşan, L.: Inteligenţă artificială - Sisteme inteligente.

[6] Dosoftei, C-tin C.: Utilizarea inteligenței computaționale în conducerea proceselor - Teză de doctorat, (2009).

[7] Ghindeanu Colhon, M.: Elemente de Logică Fuzzy.(2012).

[8] https://www.parallax.com/

[9] Mărgăritescu, M., Moldovanu, Al., Boeriu, P., Rolea, A.M.: Ultrasonic Measuring System for Deposition of Sediments in Reservoirs. International Conference 2nd International Conference on Innovations, Recent Trends and Challenges in Mechatronics, Mechanical Engineering and New High-Tech Products Development MECAHITECH'11, Bucharest, (2011).

[10] Tutorial Matlab, Comparison of Sugeno and Mamdani Methods, (2010).

[11] Tutorial Matlab, ANFIS and the ANFIS Editor GUI, (2010). 